EFEITOS DO CAPITAL INTELECTUAL SOBRE O DESEMPENHO FINANCEIRO EM EMPRESAS BRASILEIRAS E CHILENAS

\author{
EFFECTS OF INTELLECTUAL CAPITAL ON THE FINANCIAL PERFORMANCE \\ IN BRAZILIAN COMPANIES AND CHILEAN
}

\title{
EFEITOS HACEN EL CAPITAL INTELECTUAL SOBRE O DESEMPENHO FINANCEIRO EM EMPRESAS BRASILEIRAS E CHILENAS
}

\section{Salete Turra}

Graduada em Ciências Contábeis pela

Faculdade Empresarial de Chapecó

sallete.turra@gmail.com

\section{Danielle Paná Vergini}

Graduada em Matemática pela Universidade

Regional de Blumenau (FURB)

danielle_vergini@hotmail.com

\section{Fellipe André Jacomossi}

Graduado em Ciências Contábeis pelo

Centro Universitário de Brusque (UNIFEBE);

Graduado em Direito pelo UNIFEBE

fellipejacomossi@gmail.com

\section{Nelson Hein}

Doutor em Engenharia de Produção pela

Universidade Federal de Santa Catarina;

Professor do Departamento de Pós Graduação

em Ciências Contábeis da FURB

hein@furb.br

\author{
Contextus \\ ISSNe 2178-9258 \\ Organização: Comitê Científico Interinstitucional \\ Editor Científico: Marcelle Colares Oliveira \\ Avaliação : Double Blind Review pelo SEER/OJS \\ Revisão: Gramatical, normativa e de formatação \\ Recebido em 02/10/2014 \\ Aceito em 30/03/2015 \\ $2^{\text {a }}$ versão aceita em 22/04/2015 \\ $3^{\mathrm{a}}$ versão aceita em 01/06/2015
}

\section{RESUMO}

O objetivo da pesquisa foi verificar a influência do Capital Intelectual medido pelo Valor Agregado do Capital Intelectual (VACI) sobre o desempenho financeiro em empresas brasileiras e chilenas no ano de 2013. Realizou-se uma pesquisa descritiva, documental, com abordagem quantitativa por meio da técnica estatística correlação canônica. Os dados foram coletados na base de dados da Tomnson ${ }^{\circledR}$. Os resultados do estudo demonstraram correlação canônica existente entre as variáveis que compõem o VACI com as variáveis de desempenho financeiro tanto no Brasil como no Chile. Desse modo, concluiu-se que o capital intelectual influencia significativamente o desempenho financeiro das empresas brasileiras e chilenas.

Palavras-chave: Capital intelectual. Indicadores de desempenho financeiro. VACI. 


\begin{abstract}
The objective of the research was to investigate the influence of intellectual capital measured by the Intellectual Capital Value Added (VACI) on financial performance in Brazilian and Chilean companies in 2013. We conducted a descriptive, documentary, with a quantitative approach through statistical canonical correlation technique. Data were collected in the Tomnson ${ }^{\circledR}$ database. The results of the study showed existing canonical correlation between the variables that make up the VACI with the financial performance variables in Brazil and Chile. Thus, it was concluded that the intellectual capital significantly influences the financial performance of the Brazilian and Chilean companies.
\end{abstract}

Keywords: Intellectual capital. Financial performance indicators. VAIC.

\title{
RESUMEN
}

El objetivo de la investigación fue investigar la influencia del capital intelectual medida por el Capital Intelectual sobre el Valor Añadido (VACI) sobre el desempeño financiero de las empresas brasileñas y chilenas en 2013. Se realizó un estudio descriptivo, documental, con un enfoque cuantitativo a través técnica de correlación canónica estadística. Los datos fueron recogidos en la base de datos Tomnson ${ }^{\circledR}$. Los resultados del estudio mostraron correlación canónica existente entre las variables que componen el VACI con las variables de desempeño financiero en Brasil y Chile. Por lo tanto, se concluyó que el capital intelectual influye significativamente en los resultados financieros de las empresas brasileñas y chilenas.

Palabras clave: Capital intelectual. Indicadores de desempeño financiero. VACI.

\section{INTRODUÇÃO}

Os investimentos em intangíveis são importantes para as negociações organizacionais, pois a capitalização deles é tecnologicamente viável, proporcionando relevância e aumento do desempenho financeiro e das atividades inovadoras das organizações. Desse modo, "na economia do conhecimento, caracterizada por ambientes competitivos complexos e dinâmicos, recursos baseados no conhecimento representam a verdadeira fonte de vantagem competitiva sustentável para a empresa" (LEV, 1999; PABLOS, 2003, p. 61).

Para Barrosoq e Gomes (1999), os ativos intangíveis têm grande importância para as empresas, superando muitas vezes os ativos contábeis, por isso a relação entre eles tem se tornado frequente em pesquisas no âmbito contábil, demonstrando que o conhecimento torna-se mensurável e gerenciável.

A mensuração da aprendizagem e do conhecimento está relacionada com a disseminação e incorporação das áreas e departamentos das empresas. Dessa maneira, antes da era do conhecimento, as empresas viviam no mundo dos tangíveis, o que funcionava bem com as práticas contábeis tradicionais. No entanto, no contexto contemporâneo, as organizações têm se adaptado ao estilo de gestão moderna e à contabilização dos intangíveis em resposta à competividade global 
(SILVA, 2004; $\quad$ CHAREONSUK; CHANSA-NGAVEJ, 2008).

Nessa contextura, para Janosevic, Dzenopoljac e Bontis (2013), não existe um conceito apropriado para o capital intelectual, por meio de seus benefícios e de sua dependência de recursos não materiais, pode-se definir como sua fonte a mente humana. A mensuração do capital intelectual e o seu impacto sobre o desempenho financeiro são questões que devem ser analisadas em conjunto para, assim, poder definir o modelo mais adequado e estimar os seus efeitos, bem como sua contribuição para o desempenho empresarial.

A gestão do conhecimento cria valor aos ativos intangíveis das empresas, pois se refere ao processo de transformação da informação em conhecimento gerando um melhor desempenho financeiro e valor para a companhia. Assim, "a geração de riqueza nas empresas está diretamente relacionada com os ativos intangíveis, pois esses ativos são responsáveis pelos desempenhos econômicos superiores e pela geração de valor aos acionistas" (REZENDE, 2002; PEREZ; FAMÁ, 2006).

Diante desse contexto, surgiu a seguinte pergunta de pesquisa: qual a influência do Capital Intelectual sobre o desempenho financeiro em empresas brasileiras e chilenas? O objetivo da pesquisa foi verificar a influência do Capital Intelectual, medido pelo Valor agregado do Capital Intelectual (VACI) sobre o desempenho financeiro em empresas brasileiras e chilenas no ano de 2013.

Diversos estudos anteriores também investigaram a temática capital intelectual e desempenho financeiro no contexto internacional, como: Bontis, Keow e Richardson (2000); Firer e Williams (2003); Ting e Lean (2009); Puntilho (2009); Sharabati, Jawad e Bontis (2010); Clarke, Seng e Whiting (2011); Salman, Mansor e Babatunde (2012); Aminbeidokhti e Darvishkhadem (2012); Janosevic, Dzenopoljac e Bontis (2013); Rashid Saeed, Lodhi e Iqbal (2013); Chen, Liu e Kweh (2014). Dessa maneira, o presente trabalho justifica-se e diferenciase, haja vista a não identificação de pesquisas no Brasil que relacionassem o capital intelectual especificamente com os indicadores financeiros. Para Antunes, Leite e Guerra (2007), estudar os efeitos do capital intelectual torna-se importante, pois as companhias precisam diferenciar-se em um mercado competitivo, assim analisar esses efeitos surge como um fator primordial no processo de investimento das empresas.

O estudo justifica-se ainda por estudar os países Brasil e Chile, visto que ambos apresentam uma economia estável 
na América Latina, com grandes modificações econômicas, políticas e sociais na última década (BASTOS; NAKAMURA, 2009). Além disso, apresentaram um crescimento em conjunto do PIB, no ano de 2013, de 2,5\% em relação ao ano anterior (BANCO MUNDIAL, 2014).

\section{REFERENCIAL TEÓRICO}

Na revisão de literatura, abordamse primeiramente os preceitos relativos ao capital intelectual. Na sequência, trata-se dos aspectos relativos ao desempenho financeiro. Além disso, abordam-se estudos anteriores, o que possibilita embasar a metodologia adotada neste estudo e aprimorar o debate sobre a temática proposta.

\subsection{Capital intelectual}

As empresas buscam meios de exploração estratégica, pelos quais possam identificar, conhecer e analisar recursos que sejam considerados superiores, com o intuito de obter vantagens competitivas e espaço no mercado. Desse modo, "na economia do conhecimento, o conhecimento tornou-se o recurso estratégico para as empresas concorrentes em ambientes dinâmicos" (PABLOS, 2003 p. 62).
Em uma organização tudo começa por meio de pessoas, logo a capacidade da empresa cresce por meio do conhecimento dos indivíduos. Suas técnicas, princípios e habilidades compõem o conhecimento de que a companhia necessita para realizar suas atividades. Dessa maneira, para que a organização desenvolva-se, necessita de pessoas, visto que "a fonte de geração de qualquer tipo de conhecimento ainda é o cérebro humano, segundo o estado da arte tecnológica no qual nos encontramos". Assim, o conhecimento amplia a capacidade de renovação das organizações (PEREIRA; SANTOS, 2001, p. 63).

As constatações do conhecimento e do capital intelectual compõem uma fase de evolução da gestão da informação. No século passado o conhecimento das pessoas era pouco utilizado, pois as atividades exigiam, normalmente, apenas esforço físico, portanto o conhecimento era utilizado apenas em condições desejáveis. Atualmente o diferencial entre as empresas não são mais máquinas, e $\operatorname{sim}$ o conhecimento agregado do conjunto de pessoas, conhecidos como os ativos intangíveis. Aquelas ricas de conhecimento passam a ganhar diferencial no mercado, como as de consultoria e auditoria. Nesse sentido, o capital intelectual é "o conhecimento existente em uma organização que pode ser usado para criar uma vantagem diferenciada. Quanto mais 
inteligente, sofisticada e integrada à nova economia, $[\ldots]$ mais chances a empresa terá em sobreviver e crescer" (REZENDE, 2002, p. 78).

Salienta Coelho (2004, p. 98) que a "manutenção dos conhecimentos e das práticas da organização pretende reduzir o retrabalho e a perda de conhecimentos inerentes às habilidades e às experiências dos indivíduos que compõem a força de trabalho de uma organização". O capital intelectual oferece benefícios tanto para a geração do conhecimento como para os serviços de valor agregado.

Segundo Chen, Liu e Kweh (2014, p. 22), as empresas podem obter melhores resultados por meio de investimentos em capital humano, capital estrutural e capital empregado. Alguns investimentos inovadores fazem com que elas produzam capital humano para o planejamento e para a execução inteligente. "Os investimentos em capital estrutural são considerados importantes para a melhoria da eficiência" organizacional.

O capital estrutural é a estrutura física da empresa visto que auxilia o capital humano a transferir o conhecimento composto pelo desenvolvimento de novas tecnologias. Investindo em capital intelectual, humano e estrutural, melhorase o desempenho das organizações tanto em quantidade como em qualidade (LLORENTE; ARECHAVALA， 2008).
Compõe o capital estrutural tudo o que estiver relacionado ao conhecimento nas organizações como banco de dados, organogramas, manuais de processos, rotinas em que o valor deles é superior ao valor material (BONTIS; KEOW; RICHARDSON, 2000).

Os ativos de propriedade intelectual possibilitam desenvolver estruturas de ativos corporativos e de ativos de infraestrutura, em vista disso, o capital humano é utilizado intensamente para compensar os investimentos em infraestrutura. "O capital empregado engloba a eficiência que o capital estrutural e humano não consegue captar" (CLARKE; SENG; WHITING, 2011, p. 10). Janosevic, Dzenopoljac e Bontis (2013) comentaram que o capital empregado são os investimentos que foram empregados em uma empresa, composto pelos ativos líquidos.

Segundo Ciprian et al. (2012, p. 707), “o capital humano é o valor total das habilidades da equipe e se concentra competências (conhecimentos, habilidades, talentos e conhecimentos), atitude (comportamento, satisfação, motivação, desempenho e ética) e a agilidade intelectual (inovação, imitação, adaptação)".

Percebe-se o quanto os ativos intangíveis são importantes para as organizações, porém as demonstrações 
financeiras não os evidenciam de forma mais clara, visto que a contabilidade deles pode proporcionar ganhos coorporativos (LEV, 2003). Muitas vezes, os investimentos em ativos intangíveis não são registrados pelo fato de não haver estimativas razoáveis e dados concretos. Para que a empresa se torne competitiva na economia do conhecimento, é importante que se estabeleça como seus ativos intangíveis contribuem para o seu desempenho financeiro (CHAREONSUK; CHANSA-NGAVEJ, 2008). Por meio deste tópico, buscou-se compreender os aspectos sobre capital intelectual, parte-se na sequência para outro componente essencial ao estudo: os indicadores de desempenho financeiro.

\subsection{Indicadores de Desempenho}

\section{Financeiro}

$$
\text { Para Knight (1999), os }
$$

investimentos financeiros podem melhorar o desempenho organizacional e a vantagem competitiva, além disso, alavancando seu capital intelectual, a empresa torna-se mais controlada, o que oferece benefícios de curto prazo. Os indicadores financeiros são fundamentais para que as organizações possam medir o comportamento de suas variáveis financeiras, verificando assim a efetividade de sua gestão estratégica (FISCHMANN; ZILBER, 1999). Os ativos baseados no conhecimento ganham importância para o desempenho futuro e para os indicadores de crescimento das organizações (MALHOTRA, 2001). O ativo intangível é considerado como um meio de obter vantagem competitiva fazendo com que as entidades empenhemse em buscar alternativas para melhor medi-lo. Seu valor pode ser estimado de acordo com algumas variáveis organizacionais, como o ambiente, a estratégia, os processos e os resultados financeiros (PACE; BASSO; SILVA, 2003).

Sendo assim, os indicadores de desempenho financeiro podem ser definidos como instrumentos que se utilizam dos demonstrativos contábeis como fonte de informações codificadas em índices e indicadores para que, por meio deles, seja possível identificar o desempenho financeiro das empresas (CAMARGOS; BARBORSA，2005). É importante ressaltar que "os índices financeiros representam o sucesso da organizacional no médio prazo e, portanto, devem ser modelados e avaliados para que sejam determinados padrões e tendências de comportamento" (BELFIORE; FÁVERO; ANGELO, 2006, p. 20).

Mediante a avaliação do desempenho financeiro organizacional, podem-se analisar as relações empresariais, uma vez que esse processo consiste na 
avaliação e no julgamento de uma situação, buscando-se diferentes possibilidades de tomada de decisão. Os indicadores financeiros reverenciam os custos das atividades, a satisfação dos clientes, o aprendizado e a melhoria contínua interna das organizações (ZAGO et al., 2011).

A gestão do desempenho financeiro está constituída pela definição de expectativas da empresa, pelo seu monitoramento e pelo desenvolvimento da capacidade de classificar seu desempenho, dentre outros (FISCHMANN; ZILBER, 2009; BROADBENT; LAUGHLIN, 2009).

Portanto, os indicadores de desempenho financeiro são importantes instrumentos de gestão que proporcionam a maximização nos lucros empresarial e seu diferencial perante o mercado (TIAN; KETSARAPORN, 2013). Nesse contexto percebe-se que o desempenho organizacional é fator importante a ser analisado pelas empresas no Brasil e no mundo, visto que por meio dele as organizações podem monitorar e comparar a sua performance e se manter em um mercado competitivo. As medidas de desempenho "faz com que a decisão fique orientada por um único indicador construído a partir de várias abordagens de desempenho diferentes" (SILVA; CORRAR, 2012, p.45).

\subsection{Estudos Relacionados e constituição da hipótese de pesquisa}

A influência de fatores na determinação do capital intelectual é objeto de pesquisa na literatura contábil nacional e internacional. A pesquisa de Bontis, Keow e Richardson (2000) teve como objetivo analisar a influência do capital intelectual e seus componentes sobre o desempenho financeiro em empresas na Malásia. Os achados demonstraram que o capital intelectual e seus componentes têm influência significativa sobre o desempenho financeiro dos negócios.

Firer e Williams (2003) analisaram a eficiência do capital intelectual e seus componentes sobre o desempenho financeiro a partir das variáveis de rentabilidade, produtividade e da avaliação de mercado em empresas da África do Sul. Os resultados apontaram que o capital intelectual não influencia significativamente a rentabilidade, a produtividade e o valor de mercado, uma vez que o capital físico demonstrou-se significativo sobre o desempenho financeiro.

A pesquisa de Ting e Lean (2009) verificou a relação do capital intelectual com o desempenho financeiro no período de 1999 a 2007 em empresas da Malásia. Os resultados demonstraram que o VACI e o retorno dos ativos estão 
significativamente relacionados. A eficiência do capital humano e do capital empregado influencia significativamente o desempenho financeiro das empresas, contudo, o capital estrutural não influencia significativamente o desempenho financeiro das empresas.

Puntilho (2009) analisou a relação entre o VACI e o desempenho financeiro de 21 bancos listados na bolsa de Milão, na Itália. Os resultados indicaram que não há associação significativa entre as variáveis do VACI e o desempenho financeiro.

Sharabati, Jawad e Bontis (2010) em sua pesquisa examinaram a relação entre o desempenho financeiro e o capital intelectual - composto pelo capital humano, estrutural e relacional - de empresas farmacêuticas localizadas na Jordânia. Os achados mostraram que apenas o capital humano e o capital estrutural influenciaram significativamente o desempenho financeiro das empresas.

Clarke, Seng e Whiting investigaram o efeito do capital intelectual, composto nesse estudo pelo capital estrutural, humano e físicos, sobre o desempenho financeiro em empresas australianas no período de 2004 a 2008. Os resultados apontaram relação significativa entre o capital intelectual e o desempenho financeiro.

Salman, Mansor e Babatunde (2012) verificaram a relação do capital intelectual composto pela eficiência do capital humano, eficiência do capital estrutural e eficiência do capital empregado sobre o retorno dos ativos em empresas nigerianas. Os resultados indicaram relação positiva e significativa do capital intelectual e seus componentes sobre o desempenho financeiro.

Aminbeidokhti e Darvishkhadem (2012) analisaram, por meio de questionários aplicados em um banco localizado no Irã, a relação entre o capital intelectual e o desempenho financeiro. Os resultados demonstraram relação significativa entre o desempenho financeiro e o capital de cliente e, ainda, menor associação com o capital humano.

Rashid Saeed, Lodhi e Iqbal (2013) examinaram a relação do capital intelectual com o desempenho financeiro de empresas do setor de telecomunicações localizadas no Paquistão por meio da aplicação de questionários com os funcionários desse setor. Os achados apontaram associação positiva e significativa entre o capital intelectual e o desempenho financeiro.

Janosević, Dzenopoljac e Bontis (2013) investigaram em seu estudo a influência do capital intelectual e seus componentes no desempenho financeiro em empresas da Sérvia. Os achados indicaram que o VACI influencia significativamente o desempenho financeiro. O lucro líquido, a receita 
operacional de vendas e o lucro operacional não influenciaram significativamente o capital intelectual, porém o capital intelectual e estrutural influenciou significativamente o Retorno sobre o patrimônio Líquido (ROE) e o Retorno sobre ativo (ROA). Eles ainda demonstraram que o capital físico influenciou ROE.

O estudo de Chen, Liu e Kweh (2014) analisou o impacto do capital intelectual sobre as mudanças nos índices de produtividade em empresas da Malásia.

Os resultados indigitaram que são necessários investimentos em capital intelectual, haja vista que o VACI e seus componentes tiveram impacto significativo sobre mudanças na produtividade e, com isso, influenciaram o desempenho da empresa.

Diante dos resultados apresentados das pesquisas anteriores, percebe-se que este estudo se difere dos demais, visto que não foram encontradas pesquisas no Brasil que relacionam o capital intelectual e seus componentes aos indicadores de desempenho financeiros.

O Quadro 1 apresenta uma síntese dos principais fatores pesquisados relacionados ao capital intelectual e ao indicador de desempenho financeiro com os respectivos autores.

\begin{tabular}{|c|c|}
\hline FATORES & AUTORES \\
\hline Valor agregado & $\begin{array}{l}\text { Firer e Williams (2003); Janosevic, Dzenopoljac e Bontis (2013); } \\
\text { Chen, Liu e Kweh (2014) }\end{array}$ \\
\hline Eficiência do capital humano & $\begin{array}{l}\text { Bontis, Keow e Richardson (2000); Firer e Williams (2003); Ting e } \\
\text { Lean (2009); Puntilho (2009); Sharabati, Jawad e Bontis (2010); } \\
\text { Clarke, Seng, Whiting (2011); Salman, Mansor, Babatunde (2012); } \\
\text { Aminbeidokhti e Darvishkhadem (2012); Janosevic, Dzenopoljac e } \\
\text { Bontis (2013); Rashid Saeed, Lodhi, Iqbal (2013); Chen, Liu e Kweh } \\
\text { (2014) }\end{array}$ \\
\hline Eficiência do capital estrutural & $\begin{array}{l}\text { Bontis, Keow e Richardson (2000); Firer e Williams (2003); Puntilho } \\
\text { (2009); Sharabati, Jawad e Bontis (2010); Clarke, Seng, Whiting } \\
\text { (2010); Salman, Mansor, Babatunde (2012); Aminbeidokhti e } \\
\text { Darvishkhadem (2012); Janosevic, Dzenopoljac e Bontis (2013); } \\
\text { Rashid Saeed, Lodhi, Iqbal (2013); Chen, Liu e Kweh (2014) }\end{array}$ \\
\hline Eficiência do capital empregado & $\begin{array}{l}\text { Firer e Williams (2003); Ting; Lean (2009); Puntilho (2009); } \\
\text { Sharabati, Jawad e Bontis (2010); Clarke, Seng, Whiting (2011); } \\
\text { Salman, Mansor, Babatunde (2012); Aminbeidokhti e } \\
\text { Darvishkhadem (2012); Janosevic, Dzenopoljac e Bontis (2013); } \\
\text { Rashid Saeed, Lodhi, Iqbal (2013); Chen, Liu e Kweh (2014) }\end{array}$ \\
\hline Lucro Operacional & Janosevic, Dzenopoljac e Bontis (2013) \\
\hline Lucro Líquido & Janosevic, Dzenopoljac e Bontis (2013) \\
\hline Receitas operacionais de vendas & Janosevic, Dzenopoljac e Bontis (2013) \\
\hline Retorno sobre o PL & $\begin{array}{l}\text { Fischmann e zilber, (1999); Firer e Williams (2003); Janosevic, } \\
\text { Dzenopoljac; Bontis (2013) }\end{array}$ \\
\hline Retorno sobre o total de Ativos & $\begin{array}{l}\text { Fischmann e zilber, (1999); Firer e Williams (2003); Janosevic, } \\
\text { Dzenopoljac e Bontis (2013); Chen, Liu e Kweh (2014) }\end{array}$ \\
\hline
\end{tabular}

Fonte: Dados da pesquisa 
De acordo com o Quadro 1, destacam-se na literatura os fatores que compõem o capital intelectual: a) Valor Agregado; b) Eficiência do Capital Humano; c) Eficiência do Capital Estrutural; d) Eficiência do Capital Empregado; e) Valor Agregado do Capital Intelectual.

$\mathrm{O}$ fator valor agregado (VA) compõe-se pelo cálculo: VA = OUT - IN . As saídas (OUT) representam total de vendas. Entradas (IN) caracterizam todos os custos da gestão da empresa, exceto aqueles relacionados com os recursos humanos, que são vistos como investimentos. O cálculo do VA pode ser compreendido como um indicador da utilização de uma empresa eficiente em capital intelectual, ou seja, quanto maior o valor agregado da empresa, maior é a eficiência do capital intelectual dela. "A ideia básica por trás dessa abordagem reside na determinação da contribuição de todos os recursos da empresa (humanos, estruturais e físicos) para a criação de VA" (JANOSEVIC;

DZENOPOLJAC; BONTIS, 2013, p. 4). Desse modo, a metodologia VACI demonstra a contribuição de cada componente, tais como capital intelectual, estrutural e humano, para a criação de valor para as empresas (CHU; Chan;Wu, 2011).

\section{O fator Human Capital Efficiency} (HCE), que busca medir a eficiência do capital Humano, compõe-se pelo cálculo: $\mathrm{HCE}=\mathrm{VA} / \mathrm{HC} . \mathrm{O}$ human capital $(\mathrm{HC})$ denota o total de salários e ordenados, durante um ano fiscal. Assim, tem-se a contribuição relativa dos recursos humanos para a criação de VA (JANOSEVIC; DZENOPOLJAC; BONTIS, 2013).

O fator eficiência do capital estrutural (SCE) compõe-se pelo cálculo: $\mathrm{SCE}=\mathrm{VA} / \mathrm{SC}$, visto que o capital estrutural (SC) é composto por hardware, software, estrutura organizacional, patentes e marcas registradas (BONTIS, 2001).

$\mathrm{O}$ fator capital employed efficiency (CEE) ou eficiência de capital empregado compõe-se pelo cálculo: $\mathrm{CEE}=\mathrm{VA}$ (valor agregado) /CE. O capital empregado (CE) é constituído pelo capital já investido em uma empresa, isto é, pelos seus ativos líquidos (JANOSEVIC; DZENOPOLJAC; BONTIS, 2013).

Os fatores que compõem os indicadores de desempenho financeiro são: a) Lucro Operacional; b) Lucro Líquido; c) Receitas operacionais de vendas; d) Retorno sobre o PL; e) Retorno sobre o total de Ativos.

Para Janosevic, Dzenopoljac e Bontis (2013), alguns aspectos devem ser observados: a) Todos os componentes do modelo VACI, se aplicados de forma correta em termos de investimentos, tendem a gerar maior lucro operacional para as empresas; b) Empresas que 
possuem capital estrutural e humano eficientes tendem a ter lucros líquidos elevados; c) Quando o capital humano é utilizado de forma eficiente, a empresa conquista mais espaço no mercado perante seus concorrentes e consequentemente gera mais receitas operacionais de vendas; d) Os componentes do VACI tendem a influenciar as medias de desempenho em relação aos retornos sobre o patrimônio líquido; e e) Empresas que investem adequadamente em seu capital humano tendem a ter retornos sobre os ativos mais satisfatórios. Nesse sentido, o presente trabalho tem as seguintes hipóteses de pesquisa:

H1: Há uma correlação canônica estatisticamente significativa entre os indicadores do VACI sobre os indicadores de desempenho financeiro das empresas Brasileiras;

H2: Há uma correlação canônica estatisticamente significativa entre os indicadores do VACI sobre os indicadores de desempenho financeiro das empresas Chilenas.

Constata-se que tais indicadores contribuem para análise do capital intelectual, medido pelo VACI e, portanto, são fatores que podem influenciar a determinação do capital intelectual sobre o desempenho financeiro.

\section{METODOLOGIA}

A classificação do estudo no que se refere aos objetivos é uma pesquisa descritiva. A análise da pesquisa, por ser estatística, classifica-se como sendo um estudo quantitativo quanto à abordagem do problema.

A pesquisa caracteriza-se como descritiva, pois expõe os aspectos das empresas brasileiras e chilenas referentes ao capital intelectual e ao desempenho financeiro. Conforme Gil (2011), a pesquisa descritiva demonstra as características de determinado fenômeno, constituindo relação entre as variáveis envolvidas.

A abordagem do problema da pesquisa caracteriza-se como quantitativa, visto que tem como objetivo medir a relação entre o capital intelectual e os indicadores de desempenho financeiro. Para Richardson (1999), a pesquisa quantitativa é definida como análise de uma determinada população por meio de aplicações de técnicas estatísticas.

A população da pesquisa é composta por todas as empresas brasileiras listadas na BM\&FBovespa e por todas as empresas chilenas listadas na Bolsa Comércio de Santiago (BCS). Desse modo, a amostra da pesquisa é composta, apenas, pelas empresas que apresentaram as informações necessárias ao estudo, 
totalizando 166 empresas brasileiras e 137 empresas chilenas que expuseram os dados necessários para determinar o capital intelectual e os indicadores de desempenho financeiro no período de 2013.

Para construir a variável VACI e as variáveis de desempenho financeiro, utilizou-se a base de dados Thompson ${ }^{\circledR}$. O Quadro 3 apresenta as variáveis envolvidas na pesquisa e suas respectivas características, composições e fórmulas:

Quadro 2 - Variáveis da pesquisa

\begin{tabular}{|c|c|c|}
\hline \multicolumn{3}{|c|}{ Variáveis que compõem VACI } \\
\hline Indicadores & Legenda & Fórmula \\
\hline VA & Valor agregado & $V A=(O U T-I N)$ \\
\hline $\mathrm{HCE}$ & Eficiência do capital humano & $H C E=\left(\frac{V A}{H C}\right)$ \\
\hline SCE & Eficiência do capital estrutural & $S C E=\left(\frac{V A}{S C}\right)$ \\
\hline CEE & Eficiência do capital empregado & $C E E=\left(\frac{V A}{C E}\right)$ \\
\hline \multicolumn{3}{|c|}{ Variáveis Indicadores desempenho Financeiros } \\
\hline Indicadores & Legenda & Fórmula \\
\hline LO & Lucro Operacional & $L O L=(L O L)$ \\
\hline LL & Lucro Líquido & $L L=(L L)$ \\
\hline ROV & Receitas operacional de vendas & $R O V=(R O V)$ \\
\hline ROE & $\begin{array}{l}\text { LL - Lucro Líquido } \\
\text { PL - Patrimônio Líquido }\end{array}$ & $R O E=\left(\frac{L L}{P L}\right)$ \\
\hline ROA & $\begin{array}{l}\text { LL - Lucro Líquido } \\
\text { AT - Ativo Total }\end{array}$ & $R O A=\left(\frac{L L}{A T}\right)$ \\
\hline
\end{tabular}

Fonte: Dados da pesquisa

Os dados foram analisados por meio da apresentação da correlação canônica. Conforme Mingoti (2005, p.143), a correlação canônica é "o estudo das relações lineares entre dois conjuntos de variáveis". A sua análise tem como objetivo estudar as inter-relações entre conjuntos de múltiplas variáveis dependentes e independentes. "Assim, a correlação canônica identifica a estrutura ótima ou dimensionalidade de cada conjunto de variáveis que maximiza a relação entre conjuntos de variáveis independentes e dependentes" (HAIR et al., 2007, p. 363). 
Com as matrizes $\mathrm{Xi} \times \mathrm{j}$ e $\mathrm{Yi} \times \mathrm{j}$, em que $\mathrm{X}$ é referente às empresas em análise e $\mathrm{Y}$, as suas respectivas variáveis, foram realizadas assim todas as combinações possíveis, ou seja, VACI versus indicadores de desempenho financeiro, totalizando duas combinações: uma referente ao país chileno e outra referente ao país brasileiro. Desse modo, pretendeuse estabelecer as relações:

$a 1 x 1+a 2 x 2+a 3 x 3 \ldots+a j x j=b 1 y 1+b 2 y 2+b 3 y 3+\ldots b k y k$

Nesse sentido, tem-se o primeiro par de variáveis canônicas como sendo o par $\mathrm{U} 1=\mathrm{a} 1 \mathrm{x} 1+\mathrm{a} 2 \mathrm{x} 2+\ldots \operatorname{amxn} \mathrm{e} \mathrm{V} 1=$ $\mathrm{b} 1 \mathrm{y} 1+\mathrm{b} 2 \mathrm{y} 2+\ldots+$ bnyn, em que $\mathrm{a}=[\mathrm{a} 1$, $a 2, \ldots, a m]$ e $b=[b 1, b 2, \ldots, b n]$. Eles são vetores de constantes respectivamente escolhidos de modo que a correlação entre variáveis U1 e V1 seja máxima e tais que essas duas variáveis tenham variâncias iguais a 1 , isto é, $\operatorname{var}(\mathrm{U} 1)=\operatorname{Var}(\mathrm{V} 1)=1$. O mesmo também acontece para U2 e V2, $\mathrm{U} 3$ e V3, ...Vk e Uk, $\mathrm{k}=1,2, \ldots, \min (\mathrm{m}, \mathrm{n})$.

Para obter os vetores $a_{k}, b_{k} c_{k}$, está demonstrado na literatura (SEBER, 1984; ANDERSON, 2003 e TIMM, 2002) que se deve solucionar o seguinte sistema linear:

$\left\{\begin{array}{l}\left(\sum_{X Z} \sum_{Z Z}^{-1} \sum_{Z X}-\lambda_{k} \sum_{X X}\right) a_{k}=0 \\ \left(\sum_{Z X} \sum_{X X}^{-1} \sum_{X Z}-\lambda_{k} \sum_{Z Z}\right) c_{k}=0\end{array} \quad\right.$ e $\quad\left\{\begin{array}{l}\left(\sum_{Y Z} \sum_{Z Z}^{-1} \sum_{Z Y}-\lambda_{k} \sum_{Y Y}\right) b_{k}=0 \\ \left(\sum_{Z Y} \sum_{Y Y}^{-1} \sum_{Y Z}-\lambda_{k} \sum_{Z Z}\right) c_{k}=0\end{array}\right.$

Em que $\sum_{X X}, \sum_{Y y}$ e $\sum_{W w}$ são matrizes de variâncias de X, Y e Z; $\sum x z$, $\sum_{\mathrm{ZX}}, \quad \sum_{\mathrm{YZ}}, \quad \sum_{\mathrm{ZY}}$ são matrizes de covariâncias; e $\lambda_{\mathrm{k}}$ são o k-ésimos maiores autovalores das matrizes $\sum_{X X}^{-1} \sum_{X Z} \sum_{Z Z}^{-1} \sum_{Z X}$ e $\sum_{Y Y}^{-1} \sum_{Y Z} \sum_{Z Z}^{-1} \sum_{Z Y}$. Esses procedimentos levarão a formação de duas equações, $\mathrm{U}_{1}=\mathrm{W}_{1}$ e $\mathrm{V}_{1}=\mathrm{W}_{1}$, assim definidas:

$$
a_{1} x_{1}+a_{2} x_{2}+\cdots+a_{10} x_{10}=c_{1} z_{1}+c_{2} z_{2}+\cdots+c_{6} z_{6}
$$

e

$$
b_{1} y_{1}+b_{2} y_{2}+\cdots+b_{5} x_{5}=c_{1} z_{1}+c_{2} z_{2}+\cdots+c_{6} z_{6}
$$

Este estudo fará apenas a análise de $\mathrm{U}_{1}$ e $\mathrm{V}_{1}$ com $\mathrm{W}_{1}$, para cada caso, pois elas podem ser interpretadas como sendo índices de desempenho global. As demais latências não serão discutidas.
Quanto à inferência estatística, há um teste para verificar se as matrizes $\mathrm{X}$ e $\mathrm{Z}$ e Y e Z são, ou não, correlacionadas entre si. Contudo, esse teste somente se aplica quando os vetores são normais 
multivariados. Quando a normalidade multivariada é válida, também é possível construir testes estatísticos para avaliar a significância das variáveis canônicas. O software que possibilita a análise estatística de correlação canônica, utilizado no artigo,

$$
r=\sqrt{\frac{S Q(\text { modelo })}{S Q T o t a l(\text { corrigida })}},
$$

Em que SQ (modelo) denota a soma dos quadrados referentes ao modelo de regressão ajustado aos dados, e SQTotal é a soma dos quadrados em sua totalidade. Para os testes estatísticos, adotou-se um nível de significância de 0,05.

\section{APRESENTAÇÃo E ANÁLISE DOS DADOS}

Neste tópico, inicialmente, apresenta-se a correlação canônica, em seguida, observam-se os coeficientes das variáveis canônicas do primeiro conjunto e por fim as variáveis canônicas do segundo conjunto dos países Brasil e Chile.

A Tabela 1 apresenta o resultado obtido com o cálculo da correlação canônica entre o primeiro e segundo grupo é o pacote Statgraphics ${ }^{\circledR}$, em sua versão 5.1, esses testes já se apresentam por default.

$\mathrm{O}$ caso da correlação e determinação foi efetuado mediante o uso das fórmulas que seguem:

de variáveis no Brasil. No primeiro grupo têm-se as variáveis que compõem o VACI, as quais são: Valor Agregado; Eficiência do Capital Humano; Eficiência do Capital Estrutural; Eficiência do Capital Empregado. No segundo grupo têm-se as seguintes variáveis que compõem os indicadores de desempenho financeiro: Lucro Operacional; Lucro Líquido; Receitas Operacionais de Vendas; Retorno sobre o PL; Retorno sobre o Total de Ativos. Para poder-se analisar a correlação entre os indicadores do VACI e os indicadores de desempenho financeiro das empresas, realizaram-se duas combinações, uma referente às empresas brasileiras e outra referente às empresas chilenas.

Tabela 1 - Correlação canônica do Brasil

\begin{tabular}{lcclcccc}
\hline Número & $\begin{array}{c}\text { Eigen } \\
\text { Value }\end{array}$ & $\begin{array}{c}\text { Correlação } \\
\text { Canônica }\end{array}$ & $\begin{array}{l}\text { Lambda } \\
\text { Wilks }\end{array}$ & $\begin{array}{c}\text { de Qui } \\
\text { Quadrado }\end{array}$ & $\begin{array}{c}\text { G. } \\
\text { Lib. }\end{array}$ & $\boldsymbol{p}$-valor \\
\hline 1 & 0,9847 & 0,9923 & 0,0110 & 722,2680 & 20 & 0,0000 \\
2 & 0,2492 & 0,4992 & 0,7155 & 53,5581 & 12 & 0,0000 \\
3 & 0,0461 & 0,2147 & 0,9531 & 7,6896 & 6 & 0,2617 \\
4 & 0,0008 & 0,0291 & 0,9992 & 0,1358 & 2 & 0,9344 \\
\hline
\end{tabular}

Fonte: Dados da pesquisa. 
A Tabela 1 demonstra as colunas do autovalor e o teste de lambda wilks que evidenciam a matriz de covariância de cada variável responsável pela correlação. Desse modo, por meio da Tabela 1, tem-se a correlação da primeira combinação linear entre as variáveis do primeiro conjunto e as do segundo conjunto evidenciando um coeficiente de correlação de 0,9923. Dessa maneira, conforme Hair et al. (2005), a associação é alta. Além disso, a primeira combinação linear apresenta p-value ao nível de 5\%. Para este estudo, são utilizados apenas os coeficientes descritos na primeira coluna, visto que eles representam a primeira combinação linear, que proporciona maior correlação dentre as quatro combinações lineares calculadas nos relacionados países. Portanto, aceita-se a hipótese 1 de que há uma correlação significativa entre o conjunto de variáveis do VACI e as variáveis de desempenho financeiro nas empresas brasileiras.

A Tabela 2 demonstra coeficientes das variáveis canônicas do primeiro conjunto para variáveis canônicas do segundo conjunto das empresas brasileiras.

Tabela 2 - Coeficientes para as variáveis canônicas do Brasil

\begin{tabular}{|c|c|c|c|c|c|}
\hline \multirow[b]{2}{*}{ Grupos } & \multirow[b]{2}{*}{ Variáveis } & \multicolumn{4}{|c|}{ Combinações Lineares } \\
\hline & & 1 & 2 & 3 & 4 \\
\hline \multirow{5}{*}{$\begin{array}{l}\text { Fatores } \\
\text { financeiro }\end{array}$} & LL & $-0,0011$ & $-0,2512$ & 0,3642 & $-0,2998$ \\
\hline & LO & 0,1004 & 0,3928 & $-1,2680$ & 0,6016 \\
\hline & ROA & 0,3127 & 2,2157 & 0,8692 & $-0,0865$ \\
\hline & ROE & $-0,0124$ & $-0,1836$ & $-0,4618$ & $-0,9132$ \\
\hline & ROV & 0,6271 & $-2,4492$ & 0,2107 & $-0,4194$ \\
\hline \multirow{4}{*}{ Fatores do capital intelectual } & CEE & 0,0077 & $-0,9478$ & 0,3271 & 0,0205 \\
\hline & $\mathrm{HCE}$ & $-0,0014$ & $-0,4960$ & $-1,3246$ & 0,0548 \\
\hline & SCE & $-0,0035$ & 0,0119 & 0,0485 & 0,9994 \\
\hline & VA & 1,0004 & 0,4205 & 0,9130 & $-0,0152$ \\
\hline - $\quad$ Correlação & & 0,9923 & 0,4992 & 0,2147 & 0,0291 \\
\hline
\end{tabular}

Fonte: Dados da pesquisa.

Mediante a Tabela 2, pode-se observar que os coeficientes canônicos correspondentes ao primeiro par apresentam uma tendência observada (confirmada): quanto maiores as Receitas Operacionais de Vendas -0,6271 -, maior é a relação do Valor Agregado - 1,0004 -, visto que essas variáveis foram as que apresentaram maior influência se comparado com as outras variáveis do modelo. Desse modo, são determinantes fortemente relacionados, sendo comprovadas a forte correlação e a significância dos fatores.

Ainda por meio da Tabela 2, podese analisar que os coeficientes canônicos 
correspondentes ao primeiro par apresentam uma tendência observada (confirmada): quanto maior o Lucro Operacional (LOP) - 0,1004 -, menor a Eficiência do Capital Humano (HCE) 0,0014. Além disso, verificou-se que quanto menor o Lucro Líquido (LIQ) 0,0011 - e o retorno do patrimônio líquido (ROE) - -0,0124 -, maior é a eficiência do Capital Empregado (CEE) - 0,0077. Também se constata que quanto maior o Retorno do Ativo (ROA) - 0,3127 -, menor é a eficiência do capital estrutural (SCE) 0,0035 .

Nesse contexto, conforme Janosevic, Dzenopoljac e Bontis (2013), pode-se afirmar que todos os recursos da empresa: humanos, estruturais e físicos contribuem para a criação do VA. Assim, conclui-se, pela alta porcentagem do VA e pela relação com a Receita Operacional de Vendas, que as empresas brasileiras tendem a fazer mais investimentos em recursos físicos, humanos e estruturais, em vista disso, elas são propensas a serem eficientes no que diz respeito ao seu capital intelectual.

Observa-se também que quanto maiores os lucros operacionais das empresas brasileiras, elas tendem a investir menos em habilidades da equipe de funcionários que se concentram em competências, atitudes e a agilidade intelectual.

Analisa-se também que quanto menos Lucro Líquido e Retorno do Patrimônio, maior são os investimentos em Ativos Líquidos, ou seja, o emprego de capital na empresa. Além disso, constata-se que quanto maior o Retorno do Ativo, menor são os Investimentos em Estrutura Organizacional, hardware, software, patentes e marcas registradas.

A Tabela 3 apresenta o resultado obtido com o cálculo da correlação canônica entre o primeiro e segundo grupo de variáveis no Chile.

Tabela 3 - Correlação canônica do Chile

\begin{tabular}{lcclccc}
\hline Número & $\begin{array}{c}\text { Eigen } \\
\text { Value }\end{array}$ & $\begin{array}{c}\text { Correlação } \\
\text { Canônica }\end{array}$ & $\begin{array}{l}\text { Lambda } \\
\text { Wilks }\end{array}$ & $\begin{array}{c}\text { de Qui } \\
\text { Quadrado }\end{array}$ & $\begin{array}{c}\text { G. } \\
\text { Lib. }\end{array}$ & $\boldsymbol{p}$-valor \\
\hline 1 & 0,9776 & 0,9887 & 0,0012 & 884,8150 & 20 & 0,0000 \\
2 & 0,9352 & 0,9670 & 0,0521 & 387,1440 & 12 & 0,0000 \\
3 & 0,1826 & 0,4273 & 0,8029 & 28,7535 & 6 & 0,0001 \\
4 & 0,0177 & 0,1330 & 0,9823 & 2,3370 & 2 & 0,3108 \\
\hline
\end{tabular}

Fonte: Dados da pesquisa.

Por meio da Tabela 2, pode-se observar os grupos de variáveis que correspondem às empresas do Chile, as quais são o coeficiente de correlação da 
primeira combinação, que foi de $98,87 \%$; a força de associação positiva, a qual também se demonstrou muito forte; e o $p$ valor da primeira combinação linear o qual foi de 0,000 menor que o nível de 5\%, podendo afirmar a significância. A primeira coluna apresenta maior correlação entre as quatro combinações lineares. Portanto, aceita-se a hipótese 2 de que há uma correlação significativa entre o conjunto de variáveis do VACI e as variáveis de desempenho financeiro nas empresas chilenas.

A Tabela 4 demonstra os coeficientes das variáveis canônicas do primeiro conjunto para as variáveis canônicas do segundo conjunto das empresas chilenas.

Tabela 4 - Coeficientes para as variáveis canônicas do Chile

\begin{tabular}{|c|c|c|c|c|c|}
\hline \multirow[b]{2}{*}{ Grupos } & \multirow[b]{2}{*}{ Variáveis } & \multicolumn{4}{|c|}{ Combinações Lineares } \\
\hline & & 1 & 2 & 3 & 4 \\
\hline \multirow{5}{*}{$\begin{array}{l}\text { Fatores } \\
\text { desempenho } \\
\text { financeiro }\end{array}$} & LL & 0,1255 & 1,0008 & 0,1046 & $-0,0886$ \\
\hline & LO & 0,0396 & $-0,0923$ & $-1,6467$ & 0,1022 \\
\hline & ROA & 0,2466 & $-0,0810$ & 1,3219 & $-0,5135$ \\
\hline & ROE & $-0,0160$ & 0,0058 & $-0,0354$ & $-0,9914$ \\
\hline & ROV & 0,7368 & $-0,0356$ & $-0,0213$ & 0,1509 \\
\hline \multirow{4}{*}{$\begin{array}{l}\text { Indicadores do } \\
\text { capital intelectual }\end{array}$} & CEE & 0,0161 & 0,1504 & 0,0317 & 0,9892 \\
\hline & $\mathrm{HCE}$ & $-0,0544$ & 0,0047 & 1,1533 & $-0,0263$ \\
\hline & SCE & 0,1158 & 0,9763 & 0,0028 & $-0,1919$ \\
\hline & VA & 1,0141 & $-0,1632$ & $-0,5305$ & $-0,0026$ \\
\hline $\begin{array}{l}\text { R1 - Correlação } \\
\text { Canônica }\end{array}$ & & $\mathbf{0 , 9 8 8 7}$ & 0,9670 & 0,4273 & $\mathbf{0 , 1 3 3 0}$ \\
\hline
\end{tabular}

Fonte: Dados da pesquisa.

Mediante aTabela 4, pode-se constatar que os coeficientes canônicos correspondentes ao primeiro par apresentam uma tendência observada(confirmada): quanto maiores as Receitas Operacionais de Vendas - 0,7368 -, maior é a dependência do Valor Agregado - 1,0141 -, visto que essas variáveis foram as que apresentaram maior influência se comparado com as outras do modelo. Logo, são determinantes fortemente relacionados, sendo comprovadas a forte correlação e a significância dos fatores.

Ainda por meio da Tabela 2, podese analisar que os coeficientes canônicos correspondentes ao primeiro par apresentam uma tendência observada ( confirmada): quanto maior o Lucro Operacional (LOP) - 0,0396 -, menor a Eficiência do Capital Humano (HCE) 0,0544. Além disso, constatou-se que 
quanto maior o Lucro Líquido (LIQ) 0,1255 e menor o Retorno do Patrimônio Líquido (ROE) - -0,0160 -, maior é a Eficiência do Capital Empregado (CEE) 0,0161. Também se constata que quanto maior o Retorno do Ativo (ROA) - 0,2466 -, maior é a Eficiência do Capital Estrutural (SCE) 0,1158.

Nesse sentido, conforme Janosevic, Dzenopoljac e Bontis (2013), todos os recursos da empresa, humanos, estruturais e físicos contribuem para a criação do VA. Desse modo, conclui-se, pela alta porcentagem do VA e a relação com a Receita Operacional de Vendas, que as empresas chilenas tendem a fazer mais investimentos em recursos físicos, humanos e estruturais. Em vista disso, elas tendem a ser eficiente em capital intelectual.

Observa-se, ainda, que quanto maiores são os lucros operacionais das empresas brasileiras, estas tendem a investir menos em habilidades da equipe de funcionários, que se concentram nas competências (conhecimentos, habilidades, talentos e conhecimentos), na atitude (comportamento, satisfação, motivação, desempenho e ética) e na agilidade intelectual (inovação, imitação, adaptação).

Analisa-se também que quanto maior Lucro Líquido e menor o Retorno do Patrimônio, maior são os investimentos em Ativos Líquidos, ou seja, empregar capital na empresa. Além disso, constata-se que quanto maior o Retorno do Ativo, maior são os Investimentos em Estrutura Organizacional, hardware, software, patentes e marcas registradas.

Os resultados do estudo demonstraram correlação canônica existente entre as variáveis que compõem o VACI com as variáveis de desempenho financeiro tanto no Brasil como no Chile. Dessa maneira, conclui-se que o Capital Intelectual influencia significativamente o desempenho financeiro das empresas brasileiras e chilenas.

Verifica-se ainda que as empresas chilenas obtiveram lucros líquidos maiores do que as brasileiras. Além disso, constatase que as empresas chilenas tendem a utilizar os Retornos dos Ativos para fazer investimentos em Capital Estrutural, porém nas brasileiras quanto maior o Retorno dos Ativos, menor são os Investimentos em Capital Estrutural.

Nesse sentido, pode-se concluir que a criação de valor para as empresas chilenas e brasileiras é alcançada por meio da aplicação intensiva de Capital Financeiro, Humanos, Estruturais e Físicos, em vista disso elas tendem a ser eficientes em capital intelectual.

Os resultados deste estudo vão ao encontro dos resultados das pesquisas de Bontis, Keow e Richardson (2000); Sharabati, Jawad e Bontis (2010); Clarke, 
Seng e Whiting (2011); Salman, Mansor e Babatunde (2012); Aminbeidokhti e Darvishkhadem (2012); Rashid Saeed, Lodhi e Iqbal (2013); e Liu e Kweh (2014), nas quais se identificou que o capital intelectual e seus componentes têm influência significativa sobre o desempenho financeiro. Porém os resultados desta pesquisa convergem com os achados da pesquisa de Janosević, Dzenopoljac e Bontis (2013), que apontaram que a Receita Operacional de Vendas não influencia significativamente o Capital Intelectual; com a pesquisa de Firer e Williams (2003), que apontou que o Capital Intelectual não influencia significativamente o Valor de Mercado; e ainda com a pesquisa de Puntilho (2009), que sugere que não há associação significativa entre as variáveis do VACI e o desempenho financeiro.

Os resultados deste estudo, também vão ao encontro dos achados de Ting e Lean (2009), pois identificaram que o VACI e Retorno dos Ativos estão significativamente relacionados, assim como a Eficiência do Capital Humano e do Capital Empregado influenciam significativamente o desempenho financeiro das empresas, porém diverge no que se refere ao Capital Estrutural não influenciar significativamente $\mathrm{o}$ desempenho financeiro das empresas.

\section{CONSIDERAÇÕES FINAIS}

A medição e avaliação do Capital Intelectual e seu impacto sobre o desempenho financeiro são itens a serem considerados pelas empresas, pois os investimentos em capital intelectual podem garantir vantagem competitiva. Desse modo, para analisar a influência do capital intelectual sobre o desempenho financeiro, deve ser utilizada mais de uma medida, pois o coeficiente do valor agregado do capital intelectual é um meio de encontrar um padrão para medir o capital intelectual e sua contribuição para o desempenho empresarial.

Dessa forma, o objetivo da pesquisa foi verificar a influência do Capital Intelectual, medido pelo Valor Agregado do Capital Intelectual (VACI), sobre o desempenho financeiro em empresas brasileiras e chilenas no ano de 2013. Os resultados do estudo demonstraram correlação canônica existente entre as variáveis que compõem o VACI com as variáveis de desempenho financeiro tanto no Brasil como no Chile. Constatou-se também que as empresas chilenas obtiveram lucros líquidos maiores do que as empresas brasileiras.

Os achados apontam ainda que pela alta porcentagem do VA e pela relação com a receita operacional de vendas, as empresas brasileiras e chilenas tendem a fazer mais investimentos em recursos físicos, humanos e estruturais, em vista 
disso tendem a ser eficientes em capital intelectual.

Além disso, verificou-se que as empresas chilenas tendem a utilizar os Retornos dos Ativos em investimentos de Capital Estrutural, no entanto, nas empresas brasileiras, quanto maior $\mathrm{o}$ Retorno dos Ativos, menores são os investimentos em Capital Estrutural.

O desempenho financeiro nas empresas analisadas, possivelmente, é obtido pela aplicação intensiva de recursos estruturais, físicos e financeiros em capital intelectual. Dessa maneira, um bom desempenho financeiro é alcançado por meio da contribuição de todos os fatores que compõem o capital intelectual, tais como capital humano, estrutural e empregado. Desse modo, conclui-se que o capital intelectual influencia significativamente o desempenho financeiro das empresas brasileiras e chilenas.

Como limitação do estudo, destacase a medição de algo que não é físico e que não pode ser facilmente mensurado, visto que, para se definir o Capital Intelectual e os bens materiais, deve-se considerar que os seus efeitos tendem a ser imprevisíveis, uma vez que são utilizados valores contábeis. Como sugestão para futuras pesquisas, recomenda-se a aplicação da presente metodologia em anos posteriores, possibilitando-se assim a comparabilidade dos resultados no contexto nacional e internacional.

\section{PUBLICAÇÃO}

Artigo submetido ao Convibra 2014.

\section{REFERÊNCIAS}

AMINBEIDOKHTI, A.; DARVISHKHADEM, S. A study of effects of intellectual capital on organizational performance: A case study of Keshavarzi bank branches in Semnan province of Iran. Management Science Letters, v. 2, n. 3, p. 971-978, 2012.

ANDERSON, T.W. An Introduction to multivariate analysis. New York: John Wiley, 2003.

ANTUNES, M. T. P.; LEITE, R. S.; GUERRA, L. F. Divulgação das informações sobre ativos intangíveis e sua utilidade para avaliação de investimentos: um estudo exploratório baseado na percepção dos analistas de investimentos. In: Congresso USP de Controladoria e Contabilidade. 2007.

\section{BANCO MUNDIAL. World Bank national accounts data, and OECD National Accounts data files. Disponível em: \\ <http://www.worldbank.org/en/publication /global-economic- \\ prospects/data?region=LAC. $>$ Acesso em: 04 jun. 2014.}

BARROSOQ, A. C. O.; GOMES, E. B. P. Tentando entender a gestão do conhecimento. Revista de Administração Pública, v. 33, n. 2, p. 147 a 170, 1999.

BASTOS, D. D.; NAKAMURA, W. T. Determinantes da estrutura de capital das companhias abertas no Brasil, México e Chile no período 2001-2006. Revista 
Contabilidade \& Finanças-USP, v. 20, n. 50, p. 75-94, 2009.

BELFIORE, P. P.; FÁVERO, L. P. L.; DE ANGELO, C. F.. Aplicação de técnicas estatísticas multivariadas em empresas de operação logística no Brasil em função de indicadores econômico-

financeiros. Revista Eletrônica de

Administração, v. 12, n. 3, p. 1-22, 2006.

BONTIS, N. Assessing knowledge assets: a review of the models used to measure intellectual capital. International Journal of Management Reviews v.3, n.1, p. 4160, 2001.

BONTIS, N.; KEOW, W. C. C.; RICHARDSON, S. Intellectual capital and business performance in Malaysian industries. Journal of intellectual capital, v. 1, n. 1, p. 85-100, 2000.

BROADBENT, J.; LAUGHLIN, R. Performance management systems: A conceptual model. Management Accounting Research, v. 20, n. 4, p. 283295, 2009.

CAMARGOS, M. A.; BARBOSA, F. V. de. Análise do desempenho econômicofinanceiro e da criação de sinergias em processos de fusões e aquisições do mercado brasileiro ocorridos entre $1995 \mathrm{e}$ 1999. REGE Revista de Gestão, v. 12, n. 2, p. 99-115, 2005.

CHAREONSUK, C.; CHANSA-NGAVEJ, C. Intangible asset management framework for long-term financial performance. Industrial Management \& Data Systems, v. 108, n. 6, p. 812-828, 2008.

CHEN. F.C; LIU, Z. J; KWEH, Q.L . Intellectual capital and productivity of Malaysian general insurers. Economic Modelling, v. 36, n.1 p. 413-420, 2014.
CHU, S.; CHAN, K., Wu, W. Charting intellectual capital performance of the gateway to China. Journal of Intellectual Capital, v.12, n.2, p.: 249-276. 2011.

CIPRIAN, G. G.; VALENTIM, R.; LUCIA, V.M.; MADALINA, G. A.Elaboration of accounting financial report on structural capital. ProcediaSocial and Behavioral Sciences, v. 62, n.1 p. 706-710, 2012.

CLARKE, M.; SENG, D.; WHITING, R. H. Intellectual capital and firm performance in Australia. Journal of Intellectual Capital, v. 12, n. 4, p. 505530, 2011.

COELHO, E. M. Gestão do conhecimento como sistema de gestão para o setor público. Revista do serviço público, v. 55, n. 1 e 2, p. 89-115, 2004.

FIRER, S.; WILLIAMS, S. M. Intellectual capital and traditional measures of corporate performance. Journal of intellectual capital, v. 4, n. 3, p. 348-360, 2003.

FISCHMANN, A. A.; ZILBER, M. A. Utilização de indicadores de desempenho como instrumento de suporte à gestão estratégica. XXIII Encontro da Associação Nacional de Pós Graduação e Pesquisa em Administração (ANPAD).. Anais...,p.1-14 1999.

FISCHMANN, A. A.; ZILBER, M. A. Utilização de indicadores de desempenho para a tomada de decisões estratégicas: um sistema de controle. Revista de Administração Mackenzie, v. 1, n. 1, P. 10-25. 2009.

GIL, A. C. Métodos e Técnicas de Pesquisa Social. 6. ed. São Paulo: Atlas, 2011.

HAIR JR, J. F.; ANDERSON, R. E.; TATHAM, R. L.; BLACK, W. C. Análise 
multivariada de dados. 5. ed. P. 47-57. Porto Alegre: Bookman, 2005.

HAIR, J. F.; ANDERSON, R.E; TATHAM, R. L.; BLACK, W.C. Análise multivariada de dados. P.600. Porto Alegre: Bookman, 2007.

JANOSEVIĆ, S.; DZENOPOLJAC D.; BONTIS N. Intellectual Capital and Financial Performance in Serbia.

Knowledge and Process Management, v. 20, n.1, p.1-11, 2013. Disponível em: <www.wileyonlinelibrary.com. > Acesso em: 09 ago. 2014.

KNIGHT, D. J. Performance measures for increasing intellectual capital. Strategy \& leadership, v. 27, n. 2, p. 22-27, 1999.

LEV B. Remarks on the measurement, valuation, and reporting of intangible assets. FRBNY Economic Policy Review, September, n. 1, p. 17-21, 2003.

LEV, B.; ZAROWIN, P. The boundaries of financial reporting and how to extend them. Journal of Accounting research,n. 2, v. 37, p. 353-385, 1999.

\section{LLORENTE, C. M.; ARECHAVALA N.}

S. Análisis de los objetivos del mercado de trabajo y de capital humano y tecnológico de la Estrategia de Lisboa por

Comunidades Autónomas. Un indicador de cumplimiento. Cuadernos de

Economía, v. 31, n. 35 p. 65-90, 2008.

MALHOTRA, Y. Knowledge assets in the global economy: assessment of national intellectual capital. Knowledge management and business model innovation, v. 3 n.8, p. 232-249, 2001.

MINGOTI, S.A. Análise de dados através de métodos de estatística multivariada uma abordagem aplicada. Bel Horizonte: Editora UFMG, 2005.
PABLOS, P. O. de. Intellectual capital reporting in Spain: a comparative view. Journal of Intellectual Capital, v. 4, n. 1, p. 61-81, 2003.

PACE, E. S. U.; BASSO, L. F. C.; SILVA, M. A. da. Indicadores de desempenho como direcionadores de valor. Revista de Administração contemporânea, v. 7, n. 1, p. 37-65, 2003.

PEREIRA, M. I.; SANTOS, S. A. Modelo de gestão: uma análise conceitual. Pioneira Thomson Learning, 2001.

PEREZ, M. M.; FAMÁ, R. Ativos intangíveis e o desempenho empresarial. Revista Contabilidade \& Finanças, v. 17, n. 40, p. 7-24, 2006.

PUNTILLO, P. Intellectual capital and business performance. Evidence from Italian banking industry. Электронный журнал Корпоративные Финансы, v12, n4, p. 97-115, 2009.

RASHID SAEED, A. S.; LODHI, R. N.; IQBAL, A. Intellectual Capital and Organizational Performance: An Empirical Study in Telecom Sector of Pakistan. Middle-East Journal of Scientific Research, v. 18, n. 4, p. 517523, 2013.

REZENDE, Y. Informação para negócios: os novos agentes do conhecimento e a gestão do capital intelectual. Ciência da Informação, Brasília, v. 31, n. 1, p. 75-83, 2002.

RICHARDSON, R. J. Pesquisa social: métodos e técnicas. 2. ed. São Paulo: Atlas, 1999.

SALMAN, R. T.; MANSOR, M.; BABATUNDE, A.D. Impact of Intellectual Capital on Return on Asset in Nigerian Manufacturing Companies. Interdisciplinary journal of research in business, v. 2, n.4, p. 4, 2012. 
SEBER, G.A.F. Multivariate

observations. New York: John Wiley and Sons, 1984.

SHARABATI, A.-A. A.; JAWAD, S. N.; BONTIS, N. Intellectual capital and business performance in the pharmaceutical sector of Jordan. Management Decision, v. 48, n. 1, p. 105-131, 2010.

SILVA M., M. A. da; CORRAR, L. J. Análise Comparativa do Desempenho Contábil-Financeiro de Empresas com Boas Práticas de Governança Corporativa no Brasil. Revista Contabilidade e Controladoria-RC\&C, v. 4, n. 1, 2012.

SILVA, S. L da. Gestão do conhecimento: uma revisão crítica orientada pela abordagem da criação do conhecimento. Ci. Inf, v. 33, n. 2, p. 143151, 2004.

TIMM, N.H. Applied multivariate analysis. New York: Springer Verlag, 2002.

TIAN, Z.; KETSARAPORN, S.

Performance benchmarking for building best practice in business competitiveness and case study.International Journal of Networking and Virtual Organisations, v. 12, n. 1, p. 40-55, 2013.

TING, I. W. K.; LEAN, H. H. Intellectual capital performance of financial institutions in Malaysia. Journal of Intellectual capital, v. 10, n. 4, p. 588599, 2009.

ZAGO, C. A.; ABREU, L. F.;

GRZEBIELUCKAS, C.; BORNIA, A. C. Modelo de avaliação de desempenho logístico com base no Balanced Scorecard (BSC): proposta para uma pequena empresa. Revista da Micro e Pequena Empresa, v. 2, n. 2, p. 19-37, 2011. 